\title{
Chlorophyll-sensitized Photooxidation Products of Spinach Galactolipids
}

\author{
Ryo Yamauchi, Masahiro Kojima, Masatoshi IsogaI, \\ Koji KATO and Yoshimitsu UENO \\ Department of Agricultural Chemistry, Gifu University, \\ Yanagido, Gifu 501-11, Japan \\ Received May 31, 1982
}

\begin{abstract}
Spinach monogalactosyldiacylglycerol (MGDG) and digalactosyldiacylglycerol (DGDG) were oxidized with singlet molecular oxygen by the use of chlorophyll $a$ as the photosensitizer. The oxidation products were separated from the unoxidized MGDG and DGDG by reverse-phase high performance liquid chromatography (HPLC). The products separated by HPLC were identified to be mono- and di-hydroperoxides formed by ${ }^{1} \mathrm{O}_{2}$ oxidation of the $16: 3$ or $18: 3$ component of MGDG and DGDG. Each unsaturated fatty acid moiety in the MGDG and DGDG produced isomeric hydroperoxides in a manner similar to the corresponding fatty acid methyl ester.
\end{abstract}

Biological membranes are subjected to lipid peroxidation which leads to physiological damage in living organisms. The oxidation mechanism of membrane phospholipids by the free radical reaction and by the singlet molecular oxygen $\left({ }^{1} \mathrm{O}_{2}\right)$ reaction has been investigated. ${ }^{1 \sim 4)}$ Polyunsaturated fatty acids constituting phospholipids have been reported to produce hydroperoxides as the primary oxidation products. On the other hand, lipid compositions of plant chloroplast membranes are very different from those of other intracellular organs. Galactolipid is the major lipid class, and phospholipid is the minor component. The major lipid class of chloroplasts is monogalactosyldiacylglycerol (MGDG) and digalactosyldiacylglycerol (DGDG). In higher plant chloroplasts, MGDG and DGDG are characterized by high concentrations of the polyunsaturated fatty acids, $16: 3$ and $18: 3 .^{5,6)}$ Furthermore, chloroplasts contain a large amount of chlorophyll which acts as a sensitizer in the photooxidation of lipids. ${ }^{7,8)}$ Therefore, the galactolipids are subjected to lipid peroxidation. It has been reported that isolated chloroplasts upon illumination could undergo destructive peroxidation initiated by light absorbed in chlorophyll and that ${ }^{1} \mathrm{O}_{2}$ played an important role in the photoinactivation and photodestruction of chloroplasts. $^{9 \sim 11)}$ However, the photooxidation mechanism of galactolipids in chloroplast membranes is virtually unknown.

In this paper, chlorophyll-sensitized photooxidation was performed to isolated spinach MGDG and DGDG, and the oxidation products were isolated and characterized by high performance liquid chromatography (HPLC) which could separate different galactolipid molecular species. ${ }^{12)}$

\section{MATERIALS AND METHODS}

Materials. MGDG and DGDG were prepared from spinach leaves as described previously. ${ }^{12)}$ The fatty acid compositions of MGDG and DGDG were as follows: MGDG, $16: 0(0.2 \%), 16: 1(0.2 \%) 16: 2(0.3 \%), 16: 3$ $(25.5 \%), 18: 2(1.0 \%)$ and $18: 3(72.8 \%)$; DGDG, $16: 0$ $(4.3 \%), 16: 3(4.5 \%), 18: 1(2.0 \%), 18: 2(2.0 \%)$ and $18: 3$ $(87.2 \%)$. The principal molecular species of MGDG were $1-18: 3 / / 2-16: 3(51.1 \%)$ and $1-18: 3 / / 2-18: 3(45.0 \%)$ and those of DGDG were $1-18: 3 / / 2-18: 3(68.9 \%)$, by the HPLC analysis. ${ }^{12)}$ The percentages of other molecular species were each less than $3 \%$ for MGDG and $12 \%$ for DGDG, respectively. Chlorophyll $a$ was prepared from spinach leaves. ${ }^{13)}$ Lipase from Rhizopus delemer (600 U/

Abbreviations: 16:0, palmitic acid; $16: 3,7,10,13$-hexadecatrienoic acid; $18: 1$, oleic acid; $18: 3, \alpha$-linolenic acid; $16: 3 \mathrm{OOH}$, hydroperoxy hexadecatrienoic acid; $18: 3 \mathrm{OOH}$, hydroperoxy octadecatrienoic acid. 
mg) was purchased from Seikagaku Kogyo Co. Other reagents were of analytical grade.

Photooxidation procedure. Spinach MGDG or DGDG (each $20 \mu \mathrm{mol}$ ) was dissolved in $1.5 \mathrm{ml}$ of ethanol containing chlorophyll $a(0.14 \mu \mathrm{mol})$ as the photosensitizer. The solution in a reaction vessel was placed in a water bath at $25^{\circ} \mathrm{C}$ and shaken mechanically. The light source was a $60 \mathrm{~W}$ tungsten projection lamp at a distance of $20 \mathrm{~cm}$ from the vessel. At intervals, $25 \mu \mathrm{l}$ of the sample was dissolved in $5 \mathrm{ml}$ of ethanol, and the amount of conjugated diene was measured from the absorbance at $235 \mathrm{~nm}$. For structural analysis, $100 \mu \mathrm{mol}$ of MGDG or DGDG and $0.34 \mu \mathrm{mol}$ of chlorophyll $a$ were dissolved in $2 \mathrm{ml}$ of ethanol and irradiated for $22 \mathrm{hr}$.

Characterization of the photooxidized products. The photooxidized MGDG and DGDG were separated by HPLC on a column $(4.6 \times 250 \mathrm{~mm})$ of LiChrosorb RP-18 (Merck) using a JASCO Twincle pump system. The solvent was $95 \%$ aqueous methanol at a flow rate of $1.5 \mathrm{ml} / \mathrm{min}$. Lipids were detected by refractive index and by an absorbance at $235 \mathrm{~nm}$. For preparation purposes, large amounts (ca. $10 \mathrm{mg}$ in $100 \mu \mathrm{l}$ solvent) of the oxidation mixture could be separated for each injection.

The oxidation products separated by HPLC were dissolved in $0.5 \mathrm{ml}$ of $0.5 \mathrm{M}$ sodium methoxide in a methanol solution and were allowed to stand at room temperature for $10 \mathrm{~min}$. The oxidized fatty acyl moieties of lipids can be converted to methyl esters without considerable damage by this treatment. ${ }^{14)}$ The resulting methyl esters were analyzed by thin layer chromatography (TLC) with hexane-ethyl ether-acetic acid $(80: 30: 1, \mathrm{v} / \mathrm{v})$. Lipids were detected by spraying with $50 \% \mathrm{H}_{2} \mathrm{SO}_{4}$ followed by charring and the peroxides were detected by spraying with $5 \%$ pottasium iodide solution. Spots corresponding to fatty acid methyl ester and hydroperoxy fatty acid methyl ester on the plate were separated by preparative TLC, using Merck silica gel $\mathrm{PF}_{254}(0.5 \mathrm{~mm}$ thick) with the same solvent system. The compounds were detected by iodine vapor. The fatty acid methyl ester fractions were analyzed by gas liquid chramatography (GLC) on a Shimadzu GC-7A apparatus equipped with a glass column $(0.3 \times 200 \mathrm{~cm})$ packed with $15 \%$ DEGS on Chromosorb W, $80 \sim 100$ mesh, at $195^{\circ} \mathrm{C}$. The hydroperoxy fatty acid methyl ester fractions were characterized by gas chromatograph-mass spectrometry (GC-MS) after hydrogenation and trimethylsilylation. ${ }^{15)}$ GC-MS was carried out on a Hitachi Model M-52 apparatus equipped with a glass column $(0.3 \times 100 \mathrm{~cm})$ packed with $2 \%$ OV-1 on Chromosorb W, $80 \sim 100$ mesh and programmed from 200 to $240^{\circ} \mathrm{C}\left(4^{\circ} \mathrm{C} / \mathrm{min}\right)$, the mass spectra being recorded at an ionizing potential of $20 \mathrm{eV}$.

In another experiment, the oxidation products separated by HPLC were hydrolyzed by lipase. The conditions of lipase hydrolysis were the same as described pre- viously. ${ }^{12)}$ The resulting fatty acid and hydroperoxy fatty acid were analyzed by TLC with hexane-ethyl ether-acetic acid $(60: 40: 1, \mathrm{v} / \mathrm{v})$. Spots corresponding to fatty acid on the plate were analyzed by GLC after methylation with $5 \%$ methanolic $\mathrm{HCl}^{16)}$ Spots corresponding to hydroperoxy fatty acid were analyzed by GLC after hydrogenation, methylation and trimethylsilylation. The conditions for the GLC analysis were the same as those for the GC-MS analysis.

\section{RESULTS}

Spinach MGDG and DGDG were photooxidized in the presence of chlorophyll $a$ (Fig. 1). The major acyl moieties of MGDG and DGDG were trienoic acid, $16: 3$ and $18: 3$, which made up more than $90 \%$ of each galactolipid. Therefore, both lipids were readily oxidized within the lapse of illumination time. Little difference in reactivity was observed between the two lipids.

MGDG and DGDG were photooxidized for $22 \mathrm{hr}$, and the oxidation products were analyzed by HPLC. Figure 2 shows the elution pattern of photooxidized MGDG on HPLC. Five new peaks, M1, M2, M3, M4 and M5, were observed in addition to the starting MGDG peaks, M6 (1-18:3//2-16:3 MGDG) and M7 (1-18:3//2-18:3 MGDG). The five new peaks were fractionated and their structures

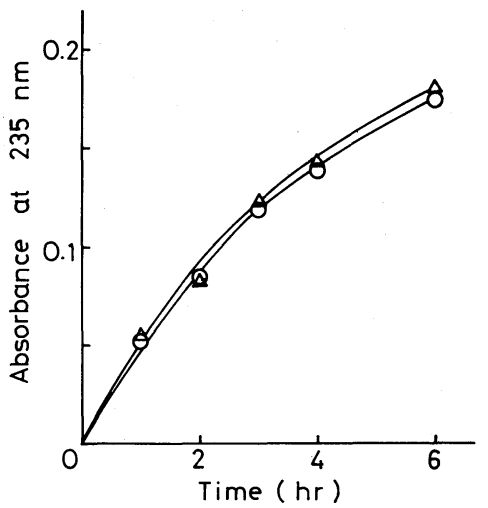

FIG. 1. Time Course of Photosensitized Oxidation of Spinach MGDG and DGDG.

The reaction mixture containing spinach MGDG or DGDG $(20 \mu \mathrm{mol})$ and chorophyll $a(0.14 \mu \mathrm{mol})$ in ethanol $(1.5 \mathrm{ml})$ was exposed to irradiation at $25^{\circ} \mathrm{C}$. $\mathrm{O}-\mathrm{O}$, MGDG; $\triangle-\triangle$, DGDG. 

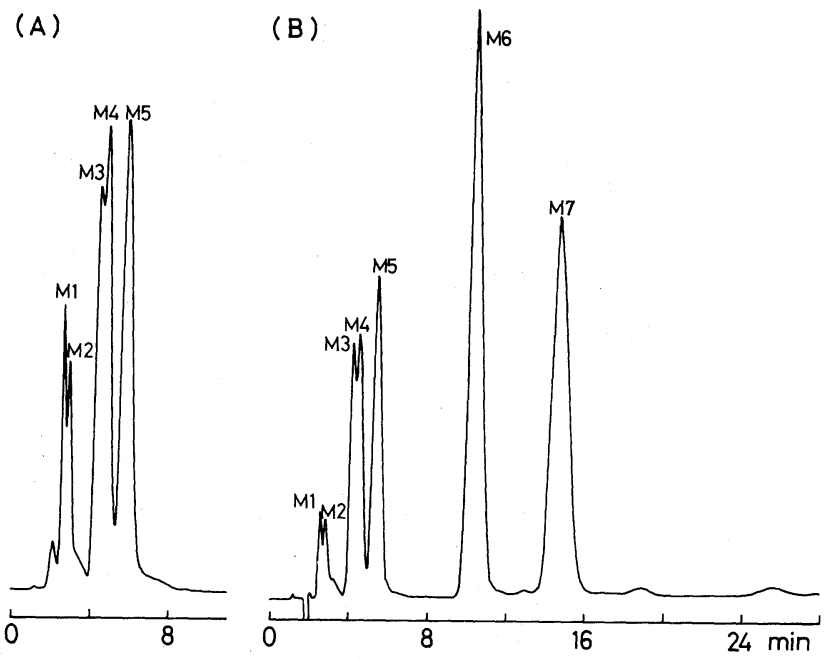

FIG. 2. High Performance Liquid Chromatogram of Photooxidized Spinach MGDG.

Detection was made by an absorbance at $235 \mathrm{~nm}$ (A) and by refractive index (B).

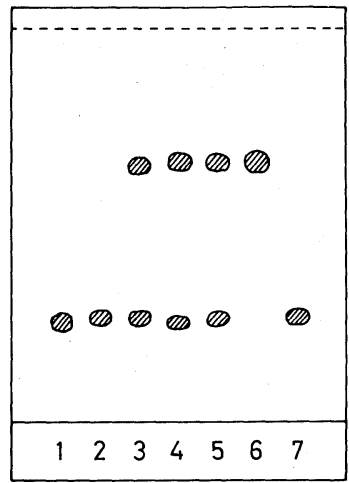

FIG. 3. Thin Layer Chromatogram of Transmethylation Products of Photooxidized MGDG.

The photooxidation products, M1, M2, M3, M4 and M5, were transmethylated with sodium methoxide in methanol solution. The resulting methyl esters of M1 (1), M2 (2), M3 (3), M4 (4) and M5 (5), methyl linoleate (6) and methyl linoleate monohydroperoxide (7) were charged on the TLC plate and developed with hexane-ethyl ether-acetic acid $(80: 30: 1, \mathrm{v} / \mathrm{v})$.

were analyzed. Each oxidation product showed a maximum in ultraviolet at $235 \mathrm{~nm}$ in ethanol, indicating the presence of a conjugated diene. Furthermore each product was shown to be a peroxide by the potassium iodide test. ${ }^{17)}$ Therefore, the photooxidation products of MGDG were assumed to be hydroperoxides.

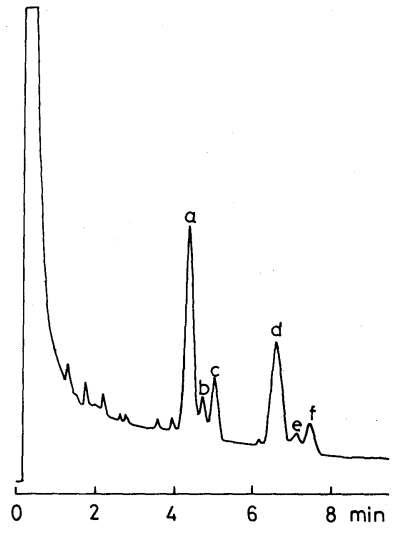

Fig. 4. Gas Chromatogram of M1 after Transmethylation, Hydrogenation and Trimethylsilylation.

The photooxidation product $\mathbf{M 1}$ indicated in Fig. 2 was transmethylated, hydrogenated and silylated for GC-MS analysis.

The products were transmethylated with sodium methoxide in methanol solution and analyzed by TLC (Fig. 3). M1 and M2 gave only one spot corresponding to hydroperoxy fatty acid methyl ester. M3, M4 and M5 gave two spots corresponding to fatty acid methyl ester and hydroperoxy fatty acid methyl ester. The spots corresponding to fatty acid methyl ester were analyzed by GLC. The fatty acid composition of each spot was 16:3 (M3), 18:3 
(M4) and 18:3 (M5), respectively. The spots corresponding to hydroperoxy fatty acid methyl ester were analyzed by GC-MS which has already been used for the analysis of hydroperoxide isomers of oxidized fatty acid methyl esters. ${ }^{15)}$ Figure 4 shows the gas chromatogram of the hydroperoxy fatty acid methyl ester of M1 after hydrogenation and trimethylsilylation. Six peaks appeared on the chromatogram. From the mass spectra, peaks $\mathrm{a}, \mathrm{b}$ and $\mathrm{c}$ were identified with hydroxy methyl hexadecanoate trimethylsilyl derivatives; $\mathrm{m} / \mathrm{z}$ $343\left(\mathrm{M}^{+}-\mathrm{CH}_{3}\right)$, and characteristic fragmentation patterns indicated that peak a was a mixture of C-7 ( $m / z 229$ and 231), C-8 $(\mathrm{m} / z 215$ and 245), C-10 (m/z 187 and 273) and C-11 $(\mathrm{m} / \mathrm{z} 173$ and 287) positional isomers of trimethylsilyloxy group; peak b was C-13 $(\mathrm{m} / \mathrm{z}$ 145 and 315$)$ and peak $c$ was C-14 $(m / z 131$ and 329) positional isomer, respectively. Peaks $\mathrm{d}$, e and f were identified to be hydroxy methyl octadecanoate trimethylsilyl derivatives from their mass spectra; $m / z 371\left(\mathrm{M}^{+}-\mathrm{CH}_{3}\right)$, and characteristic fragmentation patterns indicated that peak $d$ was a mixture of C-9 $(\mathrm{m} / z 229$ and 259), C-10 ( $m / z 215$ and 273), C-12 (m/z 187 and 301) and C-13 (m/z 173 and 315), peak e was C-15 ( $m / z 145$ and 343$)$ and peak f was C$16(\mathrm{~m} / z 131$ and 357$)$ positional isomer of the trimethylsilyloxy group, respectively. Among them, C-8 and C-13 positional isomers of hydroxy methyl hexadecanoate trimethylsilyl derivative and C-10 and C-15 positional isomers of hydroxy methyl octadecanoate trimethylsilyl derivative are the characteristic products of ${ }^{1} \mathrm{O}_{2}$ oxidation. ${ }^{15}$ ) The hydroperoxy fatty acid methyl ester fractions of $\mathbf{M 2}, \mathbf{M 3}$, M4 and M5 on the TLC plate were also. analyzed by GC-MS. After hydrogenation and trimethylsilylation, M2, M3 and M5 gave hydroxy methyl octadecanoate trimethylsilyl derivatives, respectively, and M4 gave hydroxy methyl hexadecanoate trimethylsilyl derivatives. The isomer distribution of these products was the same as that of M1.

The fractions of photooxidized MGDG as shown in Fig. 2 were hydrolyzed by Rhizopus delemer lipase which hydrolyzed the $\mathrm{C}-1$ po-

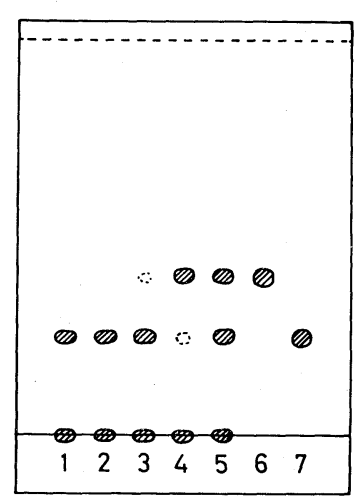

FIG. 5. Thin Layer Chromatogram of Lipase Hydrolysis Products of Photooxidized MGDG.

The photooxidized products, M1, M2, M3, M4 and M5, were hydrolyzed by lipase. The resulting fatty acid and hydroperoxy fatty acid of M1 (1), M2 (2), M3 (3), M4 (4) and M5 (5), linoleic acid (6) and linoleic acid hydroperoxide (7) were charged on the TLC plate and developed with hexane-ethyl ether-acetic acid $(60: 40: 1$, $\mathrm{v} / \mathrm{v})$.

sition fatty acid of galactolipid. ${ }^{18)}$ The resulting products were analyzed by TLC. Figure 5 shows the hydrolysis products of M1, M2, M3, M4 and M5 on the TLC plate. Spots corresponding to fatty acid and hydroperoxy fatty acid appeared on the plate. Each spot corresponding to fatty acid was identified to be 18:3 by GLC after methylation. Each spot corresponding to hydroperoxy fatty acid was determined by GLC after hydrogenation, methylation and trimethylsilylation, and was identified to be hydroxy methyl octadecanoate trimethylsilyl derivatives.

From the above data, the compound of each peak indicated in Fig. 2 was characterized to be as follows: M1, 1-18:30OH//2-16:30OH MGDG; M2, $1-18: 3 \mathrm{OOH} / / 2-18: 3 \mathrm{OOH}$ MGDG; M3, 1-18:3OOH//2-16:3 MGDG; M4, 1-18:3//2-16:3OOH MGDG; M5, 1$18: 3 \mathrm{OOH} / / 2-18: 3$ MGDG and $1-18: 3 / / 2$ $18: 3 \mathrm{OOH}$ MGDG.

The photooxidized DGDG was also analyzed by HPLC. As shown in Fig. 6, two new main peaks, D1 and D2, were observed in addition to the starting DGDG, D3 (1$18: 3 / / 2-16: 3 \quad$ DGDG $), \quad$ D4 $(1-18: 3 / / 2-$ $18: 3$ DGDG) and D5 (1-18:1//2-18:3 DGDG 

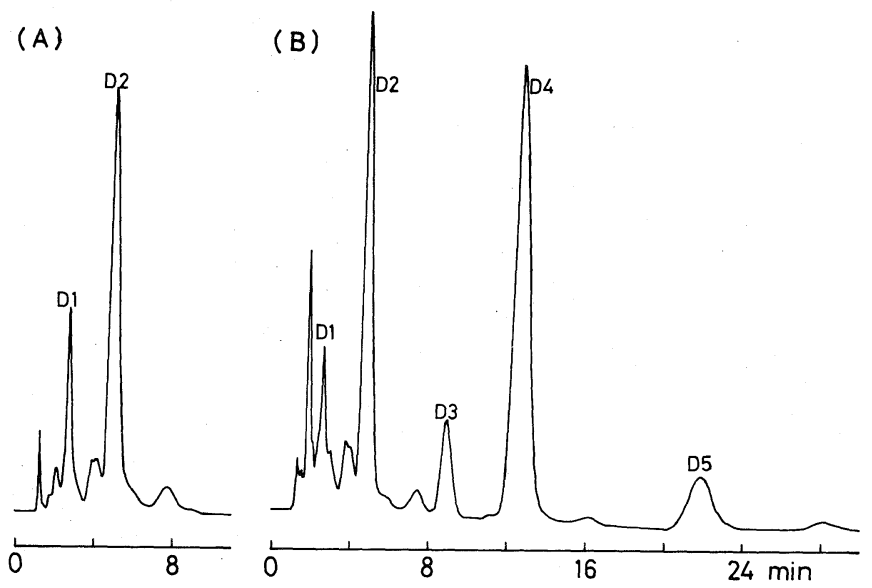

FIG. 6. High Performance Liquid Chromatogram of Photooxidized Spinach DGDG.

Detection was made by an absorbance at $235 \mathrm{~nm}$ (A) and by refractive index (B).

and 1-18:3//2-16:0 DGDG). The two peaks were fractionated and their structures were analyzed. D1 and D2 showed a maximum in ultraviolet at $235 \mathrm{~nm}$ in ethanol and were shown to be peroxides. When D1 and D2 were transmethylated with sodium methoxide, D1 gave 18:3OOH and D2 gave 18:3 and $18: 3 \mathrm{OOH}$ by the TLC and GLC analyses. The lipase hydrolysis of D1 yielded 18:30OH and that of D2 yielded $18: 3$ and $18: 3 \mathrm{OOH}$. Furthermore, the isomeric composition of $18: 3 \mathrm{OOH}$ was the same as that of the MGDG hydroperoxides. From the above data, D1 was characterized to be $1-18: 3 \mathrm{OOH} / / 2-18: 3 \mathrm{OOH}$ DGDG and D2, 1-18:3OOH//2-18: 3 DGDG and $1-18: 3 / / 2-18: 30 O H$ DGDG.

\section{DISCUSSION}

Unsaturated lipids yield isomeric monohydroperoxides when ${ }^{1} \mathrm{O}_{2}$ attacks their double bonds. ${ }^{3,15,19)}$ Thus, oxidation of spinach MGDG and DGDG with ${ }^{1} \mathrm{O}_{2}$ seems capable of forming hydroperoxides. MGDG and DGDG were found to react with ${ }^{1} \mathrm{O}_{2}$ at the position of the unsaturated fatty acid moiety to produce monohydroperoxides during photosensitized oxidation, that is, 1-18:3//2$16: 3$ MGDG gave $1-18: 3 \mathrm{OOH} / / 2-16: 3$ MGDG and 1-18:3//2-16:3OOH MGDG, 1-
$18: 3 / / 2-18: 3$ MGDG gave $1-18: 30 O H / / 2-$ $18: 3$ MGDG and $1-18: 3 / / 2-18: 3 \mathrm{OOH}$ MGDG, and 1-18:3//2-18:3 DGDG gave 1$18: 3 \mathrm{OOH} / / 2-18: 3$ DGDG and 1-18:3//2$18: 3 \mathrm{OOH}$ DGDG. Dihydroperoxides were also produced by incorporating two oxygen molecules into each unsaturated fatty acid moiety, that is, 1-18:3//2-16:3 MGDG gave 1$18: 3 \mathrm{OOH} / / 2-16: 3 \mathrm{OOH}$ MGDG $1-18: 3 / / 2-$ $18: 3 \mathrm{MGDG}$ gave $1-18: 3 \mathrm{OOH} / / 2-18: 3 \mathrm{OOH}$ MGDG, and 1-18:3//2-18:3 DGDG gave 1$18: 3 \mathrm{OOH} / / 2-18: 3 \mathrm{OOH}$ DGDG.

It appears that each unsaturated fatty acid moiety comprizing MGDG and DGDG produces isomeric hydroperoxides in a manner similar to fatty acid methyl esters. ${ }^{15)}$ Thus $16: 3$ yielded the 7-, 8-, 10-, 11-, 13- and 14-isomers and $18: 3$, the 9-, 10-, 12-, 13-, 15- and 16isomers by the reaction of spinach galactolipids with ${ }^{1} \mathrm{O}_{2}$. Therefore, the unsaturated acyl group of galactolipids is concluded to be subjected to the attack of ${ }^{1} \mathrm{O}_{2}$ in the same manner as the corresponding fatty acid methyl ester.

Lipid peroxidation in illuminated chloroplasts has been considered to be induced by the action of ${ }^{1} \mathrm{O}_{2} \cdot{ }^{9,10)}$ We detected hydroxy methyl octadecanoate isomers, which were the hydrogenated and transmethylated products of lipid hydroperoxide, in the illuminated chloro- 
plasts. ${ }^{11)}$ However, lipid hydroperoxide could not be detected in the chloroplasts. Reversephase HPLC described here can readily separate the hydroperoxides from the starting galactolipids. Therefore, the procedure reported here may make available the study of chloroplast membrane peroxidation.

\section{REFERENCES}

1) G. A. Corliss and L. R. Dugan, Jr., Lipids, 5, 846 (1970).

2) C. G. Crawford, R. D. Plattner, D. J. Sessa and J. J. Rackis, Lipids, 15, 91 (1980).

3) N. A. Porter, R. A. Wolf and H. Weenen, Lipids, 15, 163 (1980)

4) J. Terao, Y. Hirota, M. Kawakatsu and S. Matsushita, Lipids, 16, 427 (1981).

5) C. F. Allen, P. Good, H. F. Davis, P. Chisum and S. D. Fowler, J. Am. Oil Chem. Soc., 43, 223 (1966).

6) M. Nishihara, K. Yokota and M. Kito, Biochim. Biophys. Acta, 617, 12 (1980).

7) C. S. Foote, Y. G. Ching and R. M. Denny, J. Am.
Chem. Soc., 92, 5216 (1970).

8) J. Terao and S. Matsushita, Agric. Biol. Chem., 41, 2467 (1977).

9) R. L. Heath and L. Packer, Arch. Biochem. Biophys., 125, 189 (1968); R. L. Heath and L. Packer, Arch. Biochem. Biophys., 125, 850 (1968).

10) U. Takahama and M. Nishimura, Plant Cell Physiol., 16, 737 (1975); U. Takahama and M. Nishimura, Plant Cell Physiol., 17, 111 (1976).

11) R. Yamauchi and S. Matsushita, Agric. Biol. Chem., 43, 2157 (1979).

12) R. Yamauchi, M. Kojima, M. Isogai, K. Kato and Y. Ueno, Agric. Biol. Chem., 46, 2847 (1982).

13) S. Shimidzu, J. Chromatogr., 59, 440 (1971).

14) M. Morita and N. Tsushimi, Agric. Biol. Chem., 45, 1413 (1981).

15) J. Terao and S. Matsushita, J. Am. Oil Chem. Soc., 54, 234 (1977).

16) W. Stoffel, F. Chu and E. H. Ahrens, Anal. Chem., 31, 307 (1959).

17) K. Oette, J. Lipid Res., 6, 449 (1965).

18) W. Fischer, E. Heinz and M. Zeus, Hoppe-Seyler's $Z$. Physiol. Chem., 354, 1115 (1973).

19) J. Terao and S. Matsushita, Agric. Biol. Chem., 42, 667 (1978). 